\title{
Un modello Per Pirandello: Capuana e gli
} SPIRITI

\author{
Lara Michelacci*
}

ABStract: Il saggio analizza l'influenza di Capuana sull'opera di Luigi Pirandello. Dalle riflessioni sullo spiritismo, e dalle esperienze mediatiche: di scrittura automatica, Capuana elabora la teoria dell'allucinazione artistica e proclama il principio di un'arte che crea personaggi vivi, capaci di agire nella realtà come esseri autonomi. Tra il 1904 e il 1915, il giovane Pirandello raccoglierà alcuni spunti di quelle riflessioni, com'è evidente ne Il Fu Mattia Pascal, per la scena della seduta spiritica, e soprattutto nel racconto Personaggi e poi in Sei personaggi in cerca d'autore. Il mistero della percezione sensibile è il nodo comune dei due scrittori siciliani che, alle soglie del Novecento, sembrano passarsi il testimone sulle domande relative al principio della duplicità dell'essere.

PARole-chiave: Luigi Capuana; Luigi Pirandello; Scienza; Spiritismo; Personaggi

RESumo: O ensaio analisa a influência de Capuana na obra de Luigi Pirandello. Das reflexões sobre o espiritismo, e das experiências mediúnicas de escritura automática, Capuana elabora a teoria da alucinação artística e proclama o princípio de uma arte que cria personagens vivas, capazes de agir na realidade como seres autônomos. Entre 1904 e 1915, o jovem Pirandello aproveitará algumas deixas daquelas reflexões, como é evidente em $O$ falecido Mattia Pascal, pela cena da sessão espírita, e sobretudo no conto Personaggi, e depois em Seis personagens à procura de um autor. O mistério da percepção sensível é o ponto comum dos dois escritores sicilianos que, no início do século XX, parecem passar o bastão no que tange às perguntas relativas ao princípio da duplicidade do ser.

PALAVRAS-CHAVE: Luigi Capuana, Luigi Pirandello; Ciência, Espiritismo; Personagens

ABSTRACT: This essay analyses the influence of Capuana on the work of Luigi Pirandello. From the reflections on spiritualism, and from the supernatural experiences of automatic writing, Capuana elaborates the theory of artistic hallucination and proclaims the principle of an art that creates living characters, capable of acting in reality as autonomous beings. Between 1904 and 1915, the young Pirandello gathered some ideas from those reflections, as is clear in the scene of the séance from Il Fu Mattia Pascal 
and, above all, in both the short story Personaggi and in Six characters in search of an author. The mystery of sensitive perception is the common knot of the two Sicilian writers who, at the cusp of the twentieth century, seem to exchange the baton on questions related to the principle of the duality of being.

KEY-WORDS: Luigi Capuana; Luigi Pirandello; Science; Spiritualism: Characters 
Come ha notato Simona Cigliana, nel saggio del 1908 dedicato a Arte e Scienza, Pirandello sostiene che l'opera dell' artista scaturisce quasi da uno stato d'incoscienza, sul modello della condizione alterata messa in luce da Alfred Binet nel volume Les altérations de la personalité (CIGLIANA,2005, p. 103-104).

Il richiamo alla scuola di neuro-psicopatologia francese è di sicuro interesse perché pone l'accento sui cosiddetti fenomeni "metapsichici" come furono definiti da un medico e fisiologo conosciuto da Pirandello - e osservatore diretto degli esperimenti di Charcot alla Salpêtrière come Charles Richet.

Nella seconda metà dell'Ottocento, allucinazioni, condizioni psichiche alterate, trances magnetiche e spiritiche connesse agli studi sull'isteria, erano collegate alla produzione del genio, alla creazione artistica che si configura come fasi di eccitazione fuori dalla norma. Anche lo scrittore poteva essere un caso clinico da studiare insieme alle isteriche, ai sonnambuli e ai medium (BERRÈ, 2015, p. 560)

Lo stesso Cesare Lombroso nella prefazione al libro di Barzini sul caso di Eusapia Paladino rivede il giudizio negativo nei confronti dell'esperienza spiritica (Barzini, 1907) e anzi insiste sul valore conoscitivo dell'esperienza spiritica: “A questa credenza mi conforta soprattutto la scoperta che venne fatta negli ultimi tempi da Myers, Aksakov, Sergi, Hartmann di una seconda coscienza (la subliminale, l'inconscio), che percepisce ed agisce indipendentemente dai sensi e dagli organi, giungendo nella chiaroveggenza, nel sonno ipnotico, nell'estasi, nell'ispirazione geniale, spesso a risultati assai più grandi di quelli a cui approda la coscienza normale legata agli organi, ai sensi" (LOMBROSO apud BARZINI, 1907, p. 8). Il dibattito sui poteri latenti della mente doveva, di fatto, coinvolgere anche la critica letteraria e la critica estetica mettendo in primo piano il problema della creazione artistica e delle percezioni sensoriali.

D'altra parte, nel 1882 sempre Lombroso aveva pubblicato sul "Fanfulla della domenica" diretto da Capuana alcune pagine "che commenta(va)no i casi di trasposizione dei sensi nel quadro dell'isteria" (FRIGESSI, 2003, p. 347).In particolare Lombroso aveva studiato il caso di una ragazzina isterica in cui si poteva osservare la trasposizione della vista al lobo dell' orecchio, al naso, e qualche volta alla nuca; la trasposizione dell'odorato al mento, alla regione dorsale dei piedi così come il senso del gusto alla porzione interna dei femori (LOMBROSO, 1882). Un tema caro a Capuana che proprio in quegli anni elabora il suo interesse nei confronti dei fenomeni sensoriali anche sulla scorta dei lavori di Paolo Mantegazza che nel 1876 aveva pubblicato, con fotografie di Brogi, un Atlante delle espressioni del dolore in linea con gli studi di Darwin (1872).

Di tutti questi aspetti, Capuana si ricorderà in Spiritismo? (1884) dove il dibattito sul connubio tra occultismo e scienza prenderà forme più articolate anche per l'idea di un linguaggio 
che diventa forma involontaria, come nei casi di glossolalia e di scrittura automatica nello stato di trance in una sorta di "spiritualismo scientifico" (TROPEA, 2000, p. 15). In Spiritismo? Capuana riporta i casi di Fortunato Albertini, giovane incolto, ideatore di leggende e novelle sotto diretta dettatura di "una forma nebbiosa trasparente, agitata da un vento rumoroso, assai diverso dal vento ordinario, ma che non gli incute paura" (CAPUANA, 1995, p. 99) o il caso del piccolo Edoardo Gordigiani che "pel solo misterioso impulso che gli move il braccio" si profonde nella scrittura in medianità meccanica di numerose Visioni di Jacopone da Todi, la cui autenticità fu sottoposta all'esame filologico di Giosue Carducci e Alessandro D'Ancona (BALLONI, 2005, p. 74). D'altra parte Capuana prende spunto da queste osservazioni proprio per riflettere, ancora una volta, sul dato primordiale:

Visto che un semplice passaggio magnetico può togliere la coscienza della propria personalità, è da tentare se lo stesso mezzo non possa servire ad una operazione opposta, cioè a levar via gli ultimi strati di impressioni fisiologiche e psicologiche e a mettere a nudo, poco a poco, quello che ancora sussiste dentro di noi dei mondi fisiologici e psicologici estinti (CAPUANA, 1995, p. 156).

Come si vede, Capuana riflette sulla dimensione della "vita pura", una volta estinte le sovrastrutture, ed elabora una teoria sulla similarità tra il processo di scrittura e la comunicazione spiritica: quando l'artista scrive - sostiene in Spiritismo? ma anche in Per l'Arte (1885) entra in una sua particolare allucinazione analoga a quella del sonnambulismo provocato che differisce da quello delle sonnambule e delle medium soltanto "per intensità" (CAPUANA, 1995, p. 119). Un concetto simile alla creazione incosciente tipica del lavoro del genio su cui Lombroso lavorerà a lungo (CIGLIANA, 2005, p. 104). Capuana, insomma, seguendo la linea della genealogia di forme prescritte da Angelo Camillo de Meis, risolve l'ideazione artistica in un'elaborazione "intellettuale" degli elementi caduti sotto la soglia della coscienza, un segnale che occorrerà tenere a mente per tracciare le linee di congiunzione con la narrativa di Pirandello, tanto più che proprio da Camillo de Meis prende il cognome, com'è noto, il fuggiasco Mattia Pascal.

Del resto, tramite Ugo Fleres, Capuana aveva conosciuto a Roma Pirandello e si era imposto subito come maestro: al più anziano, e noto, scrittore mineolo si deve l'invito alla stesura di opere in prosa con il risultato della pubblicazione a puntate de L'Esclusa su "La Tribuna" nel 1901 (MORACE, 2002, p. 322; SIPALA, 1974, p. 9-11). Tuttavia i legami fra i due scrittori si misurano anche sulla percezione di una realtà ultrasensibile che sottende una conoscenza diversa del dato materiale, basti pensare alla scoperta dei raggi X e all'effetto sul piano letterario di una realtà ultra-sensibile (SORBELLO, 2015, p. 547-551). 
Già nel 1879 Capuana parla dello spiritismo come nuova religione dell'avvenire, religione che non ha "apostoli, ma giornali; non ha martiri ma ha le sue vittime che vanno a popolare gli ospedali di teste sconvolte da esaltamenti nervosi [...]. È una religione senza rito, senza prete, e con una certa apparenza scientifica" (CIGLIANA, 2010, p. 524). La spinta verso il meraviglioso è in Capuana fortissima tanto da prevedere nelle Cronache letterarie un'arte autonoma rispetto al mezzo, un'opera capace di superare gli ostacoli della carta, del marmo, della tela, dei colori; un'opera d'arte che si esplicherà e diventerà tangibile con la stessa rapidità del pensiero, quando insomma "le creazioni dell'intelletto immaginativo vivranno, sia pure per qualche istante, realmente fuori di noi, quasi proiettate da un cinematografo infinitamente superiore a quello inventato dai fratelli Lumière" (CAPUANA, 1899, p. 31-32).

Pirandello, del resto, doveva avere a mano questi lavori di Capuana. Nella biblioteca dello scrittore agrigentino, tra gli altri lavori di Capuana, si trovano, infatti, le Cronache letterarie, Mondo occulto e un saggio dal titolo Arte e scienza del 1904 da cui probabilmente Pirandello trae il titolo del saggio da cui siamo partiti (CIGLIANA, 2005, p. 107). In particolare Pirandello insiste sul meccanismo della creazione artistica come attività irriflessa capace di attingere dalla psicologia sperimentale ad esempio di Jean Binet. Tuttavia il legame con Capuana si fa esplicito quando Pirandello nel saggio Soggettivismo e oggettivismo nell'arte narrativa, contenuto in Arte e scienza, cita esplicitamente le riflessioni di Capuana premesse all'edizione di Coscienze del 1906:

Nella prefazione amaramente arguta a un suo recente volume di novelle, Luigi Capuana volle raffermare una sua antica convinzione: che la novella cioè dovesse unicamente esser creazione di caratteri, di personaggi che vivano nell'opera d'arte come nella realtà, per conto loro, e che la forma dovesse così intimamente esser fusa col contenuto da non doversi distinguere affatto da esso (PIRANDELLO, 1973, p. 183).

D'altra parte, come sappiamo, l'atto stesso della creazione artistica è delineato da Capuana come una prova di sospensione della realtà simile all'azione del medium.

Capuana auspica uno studio positivo dei fenomeni paranormali, studio che sarà poi realizzato, come ha osservato Silvio Balloni, da Gustav Jung nel 1902 quando pubblica a Lipsia la sua tesi di Laurea dal titolo Psicologia e patologia dei cosiddetti fenomeni occulti. Jung in questo caso analizza "le alterazioni della personalità" che portano all'estasi medianica che si configura come meccanismo simile a quello della produzione artistica (BALLONI, 2005, p. 65). Jung, infatti, sembra gettare luce retrospettiva sulle teorie di Capuana che scrive sempre in Spiritismo?(CAPUANA, 1995, p. 135):

Per quanto sia vero che la riflessione entri oggi nelle opere d'arte in maggior quantità che non nel passato, c'è sempre un punto, nell'atto della produzione, in cui la facoltà artistica agisce con completa incoscienza. Il mestiere, il tecnicismo giova, fino a un determinato grado, nell'elaborazione della forma, la prepara, la stimola, l'agevola, la mette in moto; ma l'atto, il 
vero punto della creazione si avvolge infine, come in ogni altro fenomeno vitale, nelle misteriose oscurità dell'incoscienza.

Un dato che si proietta sulla nascita dei personaggi e infatti nel 1896,e in risposta alle osservazioni di Ugo Ojetti, Capuana osserva:

Noi creiamo dei tipi! - dice lei. Peggio per loro. Il tipo è cosa astratta: è l'usuraio, ma non è Shylock; è il sospettoso, ma non è Otello; è l'esitante, il chimerizzante, ma non è Amleto, e via via [...]. L'arte, sissignore, oggi crea (quando riesce a crearli) individui, non tipi. L'artista moderno si è convinto - e a questo convincimento l'ha indotto la scienza - che ogni creatura umana è un mondo a parte, immensamente ricco, immensamente vario, quasi altrettanto infinito quanto l'universo (CAPUANA, 1898, p. 31).

Riflessioni che ritornano innegabilmente nella celebre Prefazione ai Sei personaggi in cerca d'autore, quando Pirandello insiste sul mistero della creazione artistica:

Quale autore potrà mai dire come e perché un personaggio gli sia nato dalla fantasia? Il mistero della creazione artistica è il mistero stesso della nascita naturale. [...] un artista vivendo accoglie in sé tanti germi della vita, e non può mai dire come e perché, a un certo momento, uno di questi germi vitali gli si inserisca nella fantasia per divenire anch'esso una creatura viva in un piano di vita superiore alla volubile esistenza umana (PIRANDELLO, 1965, p. 57-58).

L'esito, come vediamo, è quello della materializzazione del pensiero che Capuana affronta nell'ultima fase della sua produzione quando in Conclusione, novella della raccolta Decameroncino, i personaggi non sono solo dissociati dal loro autore ma si muovono come entità immaginative per svolgere una funzione meta-letteraria (BALLONI, 2005, p. 81).

Per Capuana sono personaggi che vivono "nell'immaginazione e nel cuore" e che pongono all'autore le ragioni della loro comparsa in scena soprattutto da un punto di vista costituzionale come riadattamento dei modelli e definizione di un paradigma narrativo. Per questa ragione evocano le sollecitazioni del periodo fiorentino, con le prove d'ipnotismo su Beppina Poggi, e con le sperimentazioni sullo spiritismo poi confluite nel Diario spiritico che riporta i casi di trance registrati dall'autore con il mezzo della scrittura intuitiva (CAPUANA, 1870). In tal modo Capuana intuisce quella "désgrégation dell'io che Poe aveva preannunciato con i suoi morbosi e patologici personaggi” (MELANI, 2006, p. 61). E intuisce che quella frammentazione passa prima di tutto dalle figure femminili che costituiscono appunto un banco di prova, una materia da dissezionare con il bisturi o sui cui fare esercizio di sperimentazione. È dunque un "romanziere che ruba il mestiere allo psicologo, al fisiologo, al professore di scienze sociali". Un romanziere capace di entrare nella pelle dei propri personaggi: "non già che predichi, che 
dimostri, che voglia far la lezione; ma egli scortica vivi i suoi personaggi; ma egli pianta il bisturi in quelle carni palpitanti con la stessa spietata indifferenza di un anatomico" (CAPUANA, 1885, p. 42-43). Cecilia, ad esempio, l'ultimo dei sei racconti di Profili di donne è in questo senso esemplare perché, pur collocandosi nella fase aurorale della produzione narrativa di Capuana, pone uno dei problemi su cui lo scrittore siciliano cercherà di riflettere per tutta la sua carriera. In una lettera a Gianformaggio del 1877 si era soffermato su un fenomeno psicologico che avrebbe costituito la ragione artistica dei Profili: "Quando saremo insieme ti racconterò a proposito di essa un fatto psicologico stranissimo che merita di essere analizzato e studiato. Essa darebbe ad un vero critico il bandolo in mano per trovare la ragione artistica dei profili”' (ZAPPULLA MUSCARÀ, 1996, p. 66). Fin dagli esordi narrativi Capuana riflette sulla percezione del reale e sulla doppia natura dell'identità. Profili di donne è infatti profondamente connotato dall'esperienza mondana e privata dell'autore ma già a quell'altezza cronologica l'assillo del giovane scrittore è volto alla riflessione sull'ambiguità dell'essere. L'incanto amoroso di Cecilia termina banalmente nel locus horribilis, dove tutto partecipa al lutto e quello che era stato lo scenario dell'amore si trasforma in spettacolo di decadenza. Tuttavia proprio quello spazio determina una riflessione indicativa:

E andando via rimuginavo:

- Ma è dunque vero che questo mondo di fuori sia una mera creazione del nostro spirito, uno scherzo, un'illusione?

Povera Cecilia! Tu non avresti mai creduto che perfino il mio rimpianto di amore sarebbe un giorno sfumato perdendosi fra le nebbie di un problema di metafisica! (CAPUANA, 1877, p. 168).

Capuana riprenderà il concetto in Spiritismo? ma è chiaro che il problema teorico si pone fin dalle pagine del Diario spiritico negli anni ' 70 quando le domande sulla scrittura, e sulla rappresentazione del reale, si confrontano con le prime prove narrative. Il problema metafisico sembra mutuato direttamente da Schopenhauer che attribuisce "l'apparizione del fantasma ad una sorta di eco dell'universo onirico sulla realtà concreta" (NAY, 1999, p. 72). Per questo motivo la percezione o illusione visiva è un tratto fondamentale: "L'occhio con cui noi vediamo i sogni può di tanto in tanto aprirsi durante la veglia" e in tal modo "ci si presentano allora delle figure che assomigliano perfettamente alle immagini giunte al cervello attraverso i sensi, tanto che sono scambiate con quelle". A tali figure "toccherà il nome di allucinazione, di visione, di seconda vista o di apparizione di spiriti” (SCHOPENHAUER, 1983, p. 373).

Capuana è convinto che le ragioni di quelle stesse apparizioni si debbano collocare nell'organismo e siano di competenza di medici e pensatori. Da qui il discorso sulla normalità e la pazzia di stretta pertinenza della medicina, e il dibattito tra spiritismo e spiritualismo che invece appartiene ai pensatori. Come leggiamo in Spiritismo? (CAPUANA, 1995, p. 46-47) scienza e 
letteratura hanno la stessa funzione euristica e rimandato al concetto di anormalità come esagerazione del fatto normale ascrivibile alla fisiologia di Claude Bernard.

Il problema dell'illusione è centrale nell'opera di Capuana, soprattutto se si tiene conto che proprio al meccanismo ottico, nelle sue manifestazioni estreme, si affida l'interpretazione del processo di scrittura: "L'allucinazione artistica" -sostiene Capuana in Spiritismo? - è il corrispettivo dell'“allucinazione sonnambolica e medianica" e determina una vera "sospensione della personalità" (CAPUANA, 1995, p. 119-121) che permette la nascita del personaggio e dunque la possibilità dell'arte.

Nel saggio Arte e scienza del 1908, da cui siamo partiti, Pirandello parla di un'attività inconscia creatrice di caratteri, di "personaggi che vivono nella realtà per conto loro", "forme" (PIRANDELLO, 1906) in cui si avvera la forma dell'opera secondo quel processo di "emancipazione dei personaggi che aveva già elaborato nella novella omonima del 1906" (CIGLIANA, 2005, p. 108).Come ha osservato Simona Cigliana (2005, p. 109), nella novella Personaggi Pirandello cita il Piano astrale di Leadbeater, la bibbia di chi si occupava di fenomeni psichici, tradotta in Italia nel 1905, e il protagonista Leandro Scoto parla di "esseri viventi che appena formati non sono più sotto il controllo del suo creatore" (PIRANDELLO, 1906, p. 1477). Nella riflessione che va dal 1904 al 1915, dal Fu Mattia Pascal a Colloquii con i Personaggi, Pirandello tornerà più volte sulla questione di queste "forme ideali" che rendono l'autore un semplice spettatore. Un dialogo fra i due scrittori siciliani che sembra maturare fecondamente sul piano narrativo quando, abbandonato l'impianto tassonomico di Lombroso, la fisiognomica comincia a prendere connotazioni diverse e "più che alla fisica appartiene alla metafisica, e deposita sul viso segni, richiami, avatars che, come rivelazioni, trascinano gli oggetti fuori di sé" (MAZZACURATI, 1993, p. 22). Non a caso, come ha notato Ilaria Muoio, è questo il periodo di mutua influenza fra i due scrittori come si nota nella similarità tra Il caso di Emilio Roxa (in Coscienze, 1905) e alcuni passaggi del Il Fu Mattia Pascal (MUOIO, 2018, p. 155).

Sono sicuramente questi gli anni di più frequente contaminazione tra i lavori di Capuana e quelli di Pirandello anche nelle prese di posizione forti su fenomeni inspiegabili come le apparizioni spiritiche. Nel 1905 lo scrittore agrigentino pubblica un saggio intitolato Un fantasma per intervenire su un fatto di cronaca riguardante la comparsa presunta o meno di spiriti misteriosi. Ora Pirandello scrive:

Come dite, signori spiriti? Dite che l'uomo ha bisogno di spiegarsi in qualche modo il mistero della morte, per trovare una norma direttrice della propria vita! Baje! Noi abbiamo la scienza, cari miei, che ci sostiene, la scienza che ci dimostra la necessità di uniformarci alle condizioni dell'esistenza [...] per tutti coloro che non sanno o non possono adattarsi, per tutti coloro che non riescono a trovare una norma direttrice nella vita, noi abbiamo già da tempo provveduto. Sicuro! Costruendo, col dovuto rispetto della scienza, bellissimi manicomi e bellissime prigioni (PIRANDELLO apud ZAPULLA 
MUSCARÀ, 1982, p. 390).

Ha già notato Simone Cigliana che il testo richiama uno dei Semiritmi di Capuana ma forse il mineolo non comprende a pieno lo spirito di Pirandello perché l'anno successivo risponde un po'stizzito con Lettera aperta a Luigi Pirandello a proposito di un fantasma (1906):

Ma come? Il problema della vita e della morte vi sembra fatuità? Veramente voi non negate i fatti, ne siete seccato. Eppure pochi anni addietro, ricordate? Eravate un gran curioso anche voi. Io non ho dimenticato la seduta del medium Politi a cui assistemmo insieme, in casa di quel principe romano del quale in questo momento mi sfugge il nome. Vedemmo cose da far strabiliare: globi fosforescenti che erravano sotto la volta dello stanzone dove si facevano gli esperimenti [...]. Il profilo di un fantasma su l'alto della tenda dietro cui stava il Politi in trance, mentre la tenda veniva spinta fin sulle nostre teste (CAPUANA apud CICLIANA, 1995, p. 240).

Ora è chiaro, e l'osservazione si deve sempre a Simona Cigliana (2005, p. 111), che di quell'esperienza ritroviamo traccia nella nota seduta spiritica del Fu Mattia Pascal e da lì derivano anche, molto probabilmente, le letture che Pirandello dimostra di aver fatto nella Casa del Granella e nel Fu Mattia Pascal: Leadbeater, Annie Besant, madame Blavatsky, Aksakov, Morselli e lo stesso Lombroso.

È dunque indubbio che da quel côté spiritico sia nata non solo l'idea della creazione artistica come processo indipendente ma anche la percezione di un dato sensoriale che può essere continuamente modificato anche a dispetto della scienza. E forse anche in questo caso uno dei modelli è l'ironia di Capuana applicata alla fotografia.

Il 3 giugno del 1881 Capuana scrive a Verga: "Ti mando due fotografie del mio laboratorio. Quel ritratto di donna dovrà farti restare con tanto di bocca aperta. Che modellatura di chiari e scuri. Se fossi romanziere come son fotografo!" (CAPUANA, 1984, p. 122).

Una nota che procede oltre il dato fotografico stesso poiché la riflessione di Capuana si appunta sempre sulla nascita e la creazione del personaggio come entità autonoma e come soggetto di un'indipendente volontà dell'autore. Sempre a proposito di Giacinta osserva di nuovo: "I veri dolori di parto li ho provati fino al $6^{\circ}$ capitolo. Quando i personaggi si furono nettamente designati ed ingaggiati nell'azione tutto andò da sé. Me li vedevo davanti come dei personaggi reali, scrivevo quasi sotto la loro dettatura" (CAPUANA, 1984, p. 72).

A conti fatti l'indagine di Capuana si pone in alternativa alla tecnica di Balzac che si vedeva accerchiato dai suoi stessi personaggi "come persone reali, impensierito dalle difficoltà di un matrimonio, attristato da una scabrosa avventura [...]” (CAPUANA, 1995, p. 225).

La posizione del Capuana-autore è tutta giocata sulla funzione creativa del 'medium' espressa in Spiritismo? dove si chiarisce che "per entrare, come sogliamo, nella pelle del nostro 
personaggio noi adoperiamo ora contrazioni muscolari e isolamenti di determinate sensazioni a fine di lasciarne libere alcune altre più confacenti al nostro scopo; ora vere interruzioni o sospensioni della nostra personalità" (CAPUANA, 1995, p. 137).

Si va dunque affermando una concezione artistica che sublima la realtà dell'atto creativo e impone nuovi principi interpretativi:

La perfetta oggettività della ben riuscita opera d'arte non ha altra origine: talché l'artista non fotografa neppure quand'egli stesso crede di soltanto fotografare. Interpretare, integrare, compire i dati più immediati della realtà con altri più complessi accumulati nel suo organismo dalle sensazioni inavvertite [...] (CAPUANA, 1995, p. 137).

In questo modo il personaggio nasce dalle proiezioni dell'autore anche nelle forme inconsce e nelle tracce mnestiche (VIOLI, 2006, p. 88), poiché "il personaggio creato è letteralmente fotografato dal corpo dello scrittore-medium, plasmato da una macchina organica [...]" (SORBELLO, 2012, p. 85).

Insomma ancora una volta "il vecchio maestro" può aver aperto le porte a quel concetto di verità su cui si gioca molta parte della narrativa di Pirandello. 


\section{Riferimenti Bibliografici}

BALLONI, S. Metapsichica e teosofia tra Capuana e Pirandello In:Antologia Vieusseux, $n^{\circ} 31$.Firenze: Gabinetto Scientifico Letterario G. P. Vieusseux, 2005, p. 63-85.

BARZINI, L. Nel mondo dei misteri con Eusapia Paladino preceduto da uno studio di C. Lombroso e seguito da molte illustrazioni medianiche. Milano: Baldini, Castoldi, 1907.

BERRÈ, A. Nemico della società: la figura del delinquente nella cultura letteraria e scientifica dell'Italia postunitaria. Bologna: Pendrangon, 2015.

CAPUANA, L. Mondo occulto.(Org. S. Cigliana.) Catania: Edizioni del Prisma, 1995. . Lettera aperta a Luigi Pirandello a proposito di un fantasma. Credenti e miscredenti dello spiritismo. In:__. Mondo occulto.(Org. S. Cigliana).Catania: Edizioni del Prisma, 1995, p. 239-242. p. $57-162$ . Spiritismo?In:__. Mondo occulto.(Org. S. Cigliana).Catania: Edizioni del Prisma, 1995, Per l'arte. Napoli: Esi, 1994.

. Comunicazioni ricevute dagli spiriti per medianità intuitiva dall'ottobre 1870 al 28 dicembre. In: CAPUANA, A. (Org). A colloquio con me stesso? Diario tra Spiritismo? e Mondo occulto. Roma: Centro Studi e Divulgazione L. Capuana, 1985, p. 11-31.

. Lettera a Giovanni Verga, 3 giugno 1881. In: RAYA, G. (Org.). Carteggio Verga-Capuana. Roma: Edizioni dell'Ateneo,1984, p. 121-123. . Profili di donne. In: __. Racconti. (Org. E. Ghidetti) Roma: Salerno, I, 1974, p. X-X. . Gli 'ismi' contemporanei (Verismo, Simbolismo, Idealismo, Cosmopolitanismo) ed altri sa-

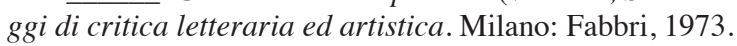

$\mathrm{V}$-XXXXII. . Nuovi ideali d'arte e di critica In: __. Cronache letterarie. Catania: Giannotta, 1899, p.

CIGLIANA, S. Spiritismo e parapsicologia nell'età positivistica. In: CAZZANIGA,G. M. (org.) Annali d'Italia: Esoterismo. Vol. 25. Torino: Einaudi, 2010, p. 521-546. 122. . Pirandello e l'ombra metafisica dei personaggi. In: Studi Italiani.Firenze, nº 2, 2005, p.103-

DARWIN, C. The Expression of the Emotions in Man and Animals. London: J. Murray, 1872.

FRIGESSI, D. Cesare Lombroso. Torino: Einaudi, 2003.

LOMBROSO, C. Studi sull'ipnotismo. 3 ed. Torino: Fratelli Bocca, 1887.

. Sull'azione del magnete e sulla trasposizione dei sensi nell'isterismo. In: . Archivio di psichiatria, antropologia criminale e scienze penali per servire allo studio dell'uomo alienato e delinquente. Vol. III, fasc.II, 1882, p. 221-337.

MANTEGAZZA, P. Atlante della espressione del dolore: fotografie prese dal vero e da molte opere d'arte che illustrano gli studi sperimentali sull'espressione del dolore. Firenze: Giacomo Brogi Fotografo Editore, 1876.

MAZZACURATI, G. Introduzione e note. In: PIRANDELLO, L. Il fu Mattia Pascal. Torino: Einaudi, 1993, p. VII-XLVIII.

MELANI, C. Effetto Poe: influssi dello scrittore americano sulla letteratura italiana. Firenze: Firenze University Press, 2006.

MORACE, A. M. Tra De Meis e Meis. In: RESTA, G. (org.) Pirandello e Napoli. Atti del Convegno di Napoli, 29 novembre-2 dicembre 2000. Roma: Salerno, 2002, p. 319-347.

MUOIO, I. Fenomenologia del naso tra Capuana e Pirandello: il caso Roxa-Pascal. In: Critica letteraria. Anno XLVI, no 178. Napoli: Paolo Loffredo Iniziative Editoriali, 2018, p. 145-158. 
NAY, L. Fantasmi del corpo, fantasmi della mente. La malattia fra analisi e racconti (1870-1900). Alessandria: Edizioni dell'Orso, 1999.

PIRANDELLO, L. Novelle per un anno. Milano: Mondadori, 1985. . Personaggi. In: __Novelle per un anno. (Org. M. Costanzo) Vol. III, Tomo 2. Milano: Mondadori, 1985, p. 1474-1479.

. Cronache stravaganti. In: ZAPPULLA MUSCARÀ, S. (Org.). Archetipi e rari del Pirandello saggista. In: Pirandello saggista. Atti del Congresso Pirandelliano. Palermo: Palumbo 1982, p. 390-391. 1973. . Opere, VI. Saggi, poesie, scritti varii. (Org. M. Lo Vecchio-Musti) Milano: Mondadori, 1965, p. 1285-1300. . Come e perché ho scritto i "Sei personaggi". In: ___. Maschere nude. Milano: Mondadori,

SCHOPENHAUER, A. Saggio sulle visioni di spiriti e su quanto vi è connesso. In Parerga e paralipomena. (Org. M. Carpitella) Milano: Adelphi, II, 1983, p. 311-420.

SIPALA, P. M. Capuana e Pirandello. Storia e testi di una relazione letteraria. Catania: Bonanno, 1974.

SORBELLO, G. Luigi Capuana e l'immaginario scientifico di fine secolo: raggi X, telepatia e la fotografia del pensiero. In: SORBELLO. G.; TRAINA, G. Dalla Sicilia a Mompracem e altro. Studi per Mario Tropea in occasione dei suoi settant' anni. Caltanissetta: Lussografica, 2015, p. 547-561.

. Iconografie veriste. Percorsi tra immagine e scrittura in Verga, Capuana e Pirandello. Acireale-Roma: Bonanno editore, 2012.

TROPEA, M. Nomi, "ethos", follia negli scrittori siciliani tra Otto e Novecento. Catania: Lussografica, 2000.

VIOLI, A. Al di là dell'utopia e del verismo.. Atti del convegno Internazionale di Studi (Trapani, 1819 settembre 2006). Marsala: La Medusa, 2008.

ZAPPULLA MUSCARÀ, S. (Org.). Luigi Capuana e le carte messaggere. Catania: C.E.C.M., 1996. . Archetipi e rari del Pirandello saggista. In: GIOVANELLI, P. D. (Org.). Pirandello saggista. Atti del Congresso Pirandelliano (Agrigento 1981). Palermo: Palumbo, 1982, p. 371-419.

Recebido: 20/06/18

Aprovado: 27/10/18 